\title{
Cancer immunotherapy for the elderly
}

Ageing has been associated with a mild pro-inflammatory milieu. Now, Bouchlaka et al. report that ageing might reinforce immune-mediated toxicity in cancer immunotherapy, as therapies that are well tolerated in young mice can be lethal in aged mice.

Most cancer immunotherapy regimens have been tested in 2-4-month old mice. This would be equivalent to treating human adolescents and young adults, but cancer very often affects the middleaged and the elderly. So, the authors tested a CD40-specific antibody and interleukin-2 (IL-2) regimen (which was previously shown to be safe and effective in young mice) in 16-month old mice. Strikingly, aged mice - whether tumour-bearing or healthy - died within 2 days of the initiation of treatment as a result of severe multi-organ pathology.
Immunotherapy-induced mortality was also observed in 9-month old, but not in 6-month old, mice and was mouse strain independent.

Treatment with CD40-specific antibodies and IL-2 markedly increased the levels of tumour necrosis factor (TNF), IL-6 and interferon- $\gamma$ in the serum of aged mice, which had higher levels of circulating pro-inflammatory cytokines than young mice even before treatment. Notably, depletion of T cells or natural killer cells did not prevent the immunotherapyinduced cytokine storm in aged mice. By contrast, depletion of macrophages protected aged mice from immunotherapy-associated lethality and liver pathology and prevented the increase in cytokine production. Consistent with these findings, the levels of TNF and IL-6 that were

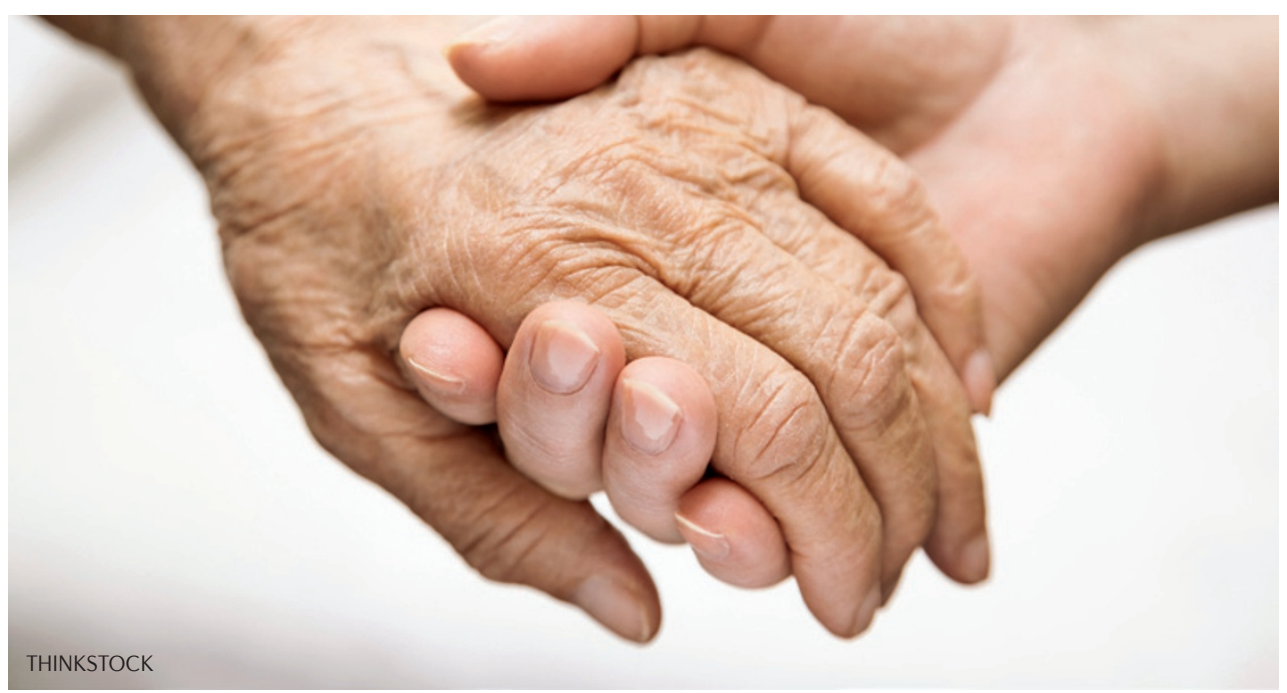

produced by macrophages from healthy humans following stimulation correlated with the donors' age, which indicates that ageing promotes the pro-inflammatory activity of macrophages. This potentially has deleterious consequences for older adults receiving immunotherapy.

But can inhibition of proinflammatory cytokines prevent the fatal consequences of immunotherapy in aged mice? When a human TNF receptor-IgG1 fusion protein (etanercept) that is approved for the treatment of autoimmune disorders in humans was administered to aged mice in combination with CD40-specific antibodies and IL-2, liver pathology and the levels of IL- 6 were reduced, leading to improved survival. Importantly, lung carcinoma-bearing aged mice receiving CD40-specific antibodies and IL-2 together with etanercept had prolonged survival, as well as enhanced $\mathrm{CD}^{+} \mathrm{T}$ cell lytic activity.

Thus, an ageing-associated pro-inflammatory milieu might predispose patients receiving immunotherapy to immune-mediated toxicity. The authors suggest that it should be investigated whether TNF blockade could also avert the immune-mediated toxicity of chemotherapy or radiotherapy in elderly and middle-aged patients.

Maria Papatriantafyllou

ORIGINAL RESEARCH PAPER Bouchlaka, M. N et al. Aging predisposes to acute inflammatory induced pathology after tumor immunotherapy. J.Exp.Med. http://dx.doi.org/10.1084/jem.20131219 (2013) 